\title{
Wahlerfolge der AfD im Osten - Reflex auf die ökonomische Lage?
}

Einigermaßen fassungslos haben die politischen Kommentatoren die jüngsten Wahlerfolge der rechtspopulistischen Partei „Alternative für Deutschland“ (AfD) insbesondere in Ostdeutschland zur Kenntnis genommen: Mit Stimmenanteilen von 24\% in Sachsen-Anhalt, 21\% in Mecklenburg-Vorpommern und immerhin 14\% in Berlin wurde das Spektrum der in den Landesparlamenten vertretenen Parteien kräftig durcheinandergewirbelt; in einigen Wahlkreisen konnte die AfD sogar die Mehrheit der Erstbzw. Zweitstimmen erreichen. Es handelt sich hierbei aber keineswegs ausschließlich um ein ostdeutsches Phänomen: Auch in Westdeutschland konnte die AfD viele Wählerstimmen auf sich ziehen (Baden-Württemberg: 15\%; Rheinland-Pfalz: 13\%), wenn auch weniger stark als in Ostdeutschland.

Vielfach werden die Wahlerfolge der AfD als reines Protestwahlverhalten gedeutet, insbesondere als Ausdruck der Unzufriedenheit mit der Flüchtlingspolitik der Bundesregierung. Tatsächlich spielt dies eine nicht unerhebliche Rolle. Eine aktuelle Studie des Deutschen Instituts für Wirtschaftsforschung (DIW) zeigt aber, dass die AfD inzwischen einen durchaus stabilen Kern an Anhängern gewinnen konnte, die sich persönlich eher am rechtskonservativen Rand des politischen Systems verorten und sich in dieser Grundeinstellung durch die „klassischen“ konservativen Parteien nicht mehr ausreichend vertreten fühlen. Insoweit ist nicht auszuschließen, dass die AfD sich auch dauerhaft in einigen deutschen Landesparlamenten etablieren wird. Dies gilt wohl vor allem für jene Länder, die von einer Koalitionsregierung unter Beteiligung von CDU und SPD geführt werden, da in einer solchen Konstellation eine Profilierung der CDU rechts von der Mitte schwieriger sein dürfte.

Gerade mit Blick auf die Wahlerfolge der AfD in Ostdeutschland liegt darüber hinaus der Gedanke nahe, den Grund hierfür in den ungünstigen wirtschaftlichen Perspektiven vieler Regionen zu suchen. Tatsächlich konnte die AfD überdurchschnittliche Stimmenanteile gerade dort verbuchen, wo die Arbeitslosigkeit hoch und die Wirtschaftskraft (und mit ihr die Löhne) eher niedrig sind. Häufig handelt es sich dabei überdies um Regionen, die durch in der Vergangenheit starke Einwohnerrückgänge gekennzeichnet waren, so dass Leistungen der öffentlichen Daseinsvorsorge immer stärker zurückgeführt werden mussten - was bei vielen Menschen das Gefühl des „Abgehängtseins“ weiter verstärkt haben dürfte. Schließlich spricht für diese Sichtweise auch, dass die AfD gerade bei Arbeitslosen (und Arbeitern) verhältnismäßig viele Wählerstimmen hat gewinnen können - bei Personengruppen also, die am wenigsten von den unstrittigen wirtschaftlichen Fortschritten seit der Vereinigung profitieren konnten.

Dennoch greift diese Erklärung zu kurz, denn auch in den wirtschaftlich prosperierenden Wahlkreisen Ostdeutschlands (und eben auch in Westdeutschland) hat die AfD hohe Stimmenanteile erhalten. Zudem zeigt die erwähnte DIW-Untersuchung, dass die Anhängerschaft der AfD sich durchaus auch aus Bevölkerungsgruppen rekrutiert, die gesellschaftlich und wirtschaftlich keineswegs als benachteiligt gelten können Personen mit wenigstens mittlerem Bildungsabschluss, Selbständige und Freiberufler, Beschäftigte mit gesichertem Einkommen und andere. Gemeinsames Merkmal der Wähler und Anhänger der AfD scheinen vielmehr vor allem eine stark ausgeprägte Unzufriedenheit mit der parlamentarischen Demokratie als solcher, die Sorge um die allgemeine wirtschaftliche Entwicklung und die eigene finanzielle Situation sowie um Kriminalität und Überfremdung der Gesellschaft durch Zuwanderung zu sein.

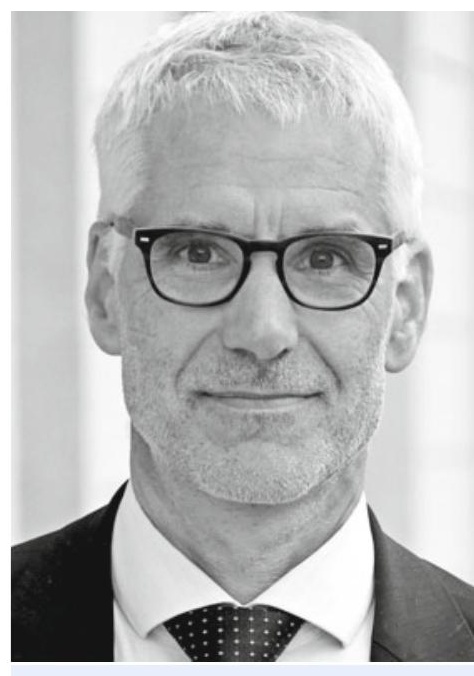

Prof. Dr. Joachim Ragnitz ist stellvertretender Geschäftsführer der Niederlassung Dresden des ifo Instituts und lehrt Volkswirtschaftslehre, insbesondere Ökonomie des Strukturwandels, an der Technischen Universität Dresden. 
Versucht man aus diesem doch eher diffusen Bild auf die eigentlichen Ursachen der Wahlerfolge der AfD zurückzuschließen, so sind es vermutlich die folgenden Erklärungsmuster, die hierfür herangezogen werden können: Zum einen das aus der Soziologie bekannte Konzept der „relativen Deprivation“, nach dem unabhängig von der objektiven Lage durch den Vergleich mit einer vermeintlich bessergestellten Bezugsgruppe ein Gefühl der subjektiven Benachteiligung entstehen kann. Gerade in Ostdeutschland, wo sich viele Menschen auch mehr als 25 Jahre nach der Vereinigung als „Bürger zweiter Klasse“ sehen, scheint hieraus ein Bedürfnis zu erwachsen, sich gegen die gesellschaftlichen und politischen Eliten zu stellen und deshalb Parteien außerhalb des gängigen Mehrheitsspektrums zu wählen.

Ein zweiter Grund dürfte sein, dass sich gerade in Ostdeutschland (aber nicht nur dort) viele Wähler durch eine ganze Reihe aktueller Entwicklungen bedroht fühlen: Viele Menschen fühlen sich durch den fortschreitenden technologischen Fortschritt und die zunehmende Globalisierung verunsichert und befürchten eine Entwertung ihrer bisherigen Lebensentwürfe - vor allem dort, wo das soziale Gefüge durch Abwanderung, instabile Beschäftigung oder Rückzug des Staates aus der Daseinsvorsorge ins Wanken geraten ist. Die starke Zuwanderung nach Deutschland, die bei vielen Überfremdungsängste auslöst - gerade in jenen Regionen, die nur geringe Erfahrungen mit dem Zusammenleben unterschiedlicher Kulturen aufweisen -, ist insoweit nur der sprichwörtliche Tropfen, der das Fass zum Überlaufen gebracht hat. Schließlich scheint es tatsächlich ein Kommunikationsproblem zwischen „den Politikern“ und „den Bürgern“ zu geben. Die Schuld hieran ist sicherlich auf beiden Seiten zu suchen - bei Politikern, die ihre Entscheidungen nicht länger erklären, aber auch bei Bürgern, die nicht bereit oder nicht in der Lage sind, Verständnis für komplizierte Zusammenhänge zu entwickeln. Die AfD (aber nicht nur sie) füllt diese Lücke mit populistischen Forderungen und vereinfachten Erklärungen, ohne dass die etablierten Parteien einen Weg gefunden hätten, diese auch als solche zu enttarnen.

Frühere Landtagswahlen gerade in den ostdeutschen Bundesländern waren vor allem durch eine geringe Wahlbeteiligung gekennzeichnet. Es scheint, dass die AfD für viele Wahlberechtigte nun ein Ventil darstellt, ihre bislang durch Wahlenthaltung offenbarte Unzufriedenheit mit dem politischen und wirtschaftlichen System Deutschlands zum Ausdruck zu bringen. Soweit diese Interpretation zutrifft, ist für die Zukunft wohl mit einer „Entzauberung“ der Partei zu rechnen: Die Erfahrungen aus denjenigen Länderparlamenten, in denen die AfD bereits länger vertreten ist, lassen nicht damit rechnen, dass sich die hohen Erwartungen ihrer Wähler in irgendeiner Weise erfüllen ließen. Aber auch das dürfte das Problem der verbreiteten Politikverdrossenheit nicht lösen, das sich gerade in Ostdeutschland wegen der auch objektiv nicht zufriedenstellenden wirtschaftlichen Entwicklung feststellen lässt. Die Notwendigkeit, sich verstärkt um strukturschwache Regionen in ganz Deutschland zu kümmern, bleibt daher auch weiterhin bestehen. Ansonsten - hierauf hat auch die Bundesregierung in ihrem jüngsten Jahresbericht zum Stand der Deutschen Einheit hingewiesen - könnte nicht nur ein weiterer Rückgang politischen und gesellschaftlichen Engagements der Menschen drohen, sondern möglicherweise auch eine zunehmende Ausbreitung

Joachim Ragnitz

ifo Institut Dresden

ragnitz@ifo.de fremdenfeindlicher Einstellungen - was gerade in Ostdeutschland, wo es aufgrund der demografischen Entwicklung auf Zuwanderung besonders ankommt, auch in wirtschaftlicher Hinsicht massive negative Auswirkungen haben würde. 